\title{
Metalenguaje y lexicografía
}

\author{
José-Álvaro Porto Dapena \\ Universidad de A Coruña
}

Desde que Jakobson ${ }^{1}$ puso de relieve la existencia de una función del lenguaje que denominó función metalingüística, consistente en la posibilidad de toda lengua natural de servir como instrumento para describirse a sí misma, característica que por cierto no corresponde a ningún otro sistema de comunicación (las señales de tráfico no se pueden estudiar mediante esas mismas señales, por ejemplo), se habla con relativa frecuencia de metalenguaje, metalengua, uso metalingüístico y metadiscurso, y en el terreno de la Lexicografía se ha llegado incluso a postular la existencia de nada menos que dos tipos de metalengua diferentes: la metalengua de contenido, o primera metalengua, y la metalengua de signo, o segunda metalengua. Pero es más: pienso que por imitación de estos términos, se han ido creado otros, que, como metateoría o metalexicografia por ejemplo, parecen indicar la presencia de una especie de "metástasis" del prefijo meta- en la terminología lingüística. Desde luego en comparación con otras funciones, como la expresiva y sobre todo la representativa, la metalingüística ha sido muy poco estudiada, y existe, a mi modo de ver, un confusionismo bastante grande que convendría aclarar de una vez por todas. Realmente los autores que se han ocupado del tema, excepción hecha de J. Rey-Debove, que le dedica toda una monografía ${ }^{2}$, lo hacen bastante sucintamente y casi siempre de una forma más bien tangencial, aunque no por ello dejen, por supuesto, de hacer interesantes aportaciones a la cuestión ${ }^{3}$.

1 Cfr. R. Jakobson (1963: 217-218).

2 Cfr. J. Rey-Debove 1978 (hay una segunda edición de Aramand Colin/Masson, Paris, 1997).

3 Me refiero, sobre todo, a E. Coseriu (1977: 107-109 y 1981: 21-22 y 293-296), J. Lyons (1980: 12-15) y S. Gutiérrez (1997: 381 y siguientes). 
En el presente trabajo me propongo, en primer lugar, hacer unas breves consideraciones acerca del metalenguaje, con el objeto de poner un mínimo de orden en las ideas acerca de esta sin duda importante cuestión, y, por otro lado, determinar de qué manera y hasta qué punto se lleva a cabo la función metalingüística en el contexto del diccionario. ¿Existen realmente en este caso dos metalenguas? ¿Hasta qué punto las definiciones lexicográficas son metalingüísticas, esto es, definiciones de las palabras, y no de las realidades por ellas representadas? ¿Existe propiamente una metalengua de definición? A éstas y otras preguntas intentaré contestar en este breve estudio.

\section{CUESTIONES GENERALES}

Antes de eso, sin embargo, vamos a intentar aclarar un poco las ideas en torno al metalenguaje en sus aspectos más generales.

\subsection{DisTINCIONES FUNDAMENTALES}

Siguiendo a Coseriu ${ }^{4}$, hay que partir ante todo de un distinción fundamental, la de metalenguaje de lengua y metalenguaje de discurso, ambos opuestos a lenguaje primario, que es el lenguaje cuyo objeto viene representado por la realidad no lingüística, frente al metalenguaje, que se ocupa a su vez del lenguaje, esto es, de la realidad lingüística. Y más concretamente, corresponde al metalenguaje de lengua el conjunto estructurado de elementos de que una lengua dispone precisamente para la descripción lingüística ${ }^{5}$. Así, por ejemplo, las mismas palabras len-

4 Cfr. E. Coseriu (1977: 107-109).

5 No hay que identificar el lenguaje primario con la lengua o sistema lingüístico, ya que en ese caso el metalenguaje de lengua vendría a ser una parte del lenguaje primario, lo que constituiría un evidente contrasentido. Lo mismo que en el metalenguaje, hay un lenguaje primario de lengua junto a un lenguaje primario de discurso, de modo que tenemos lo siguiente:

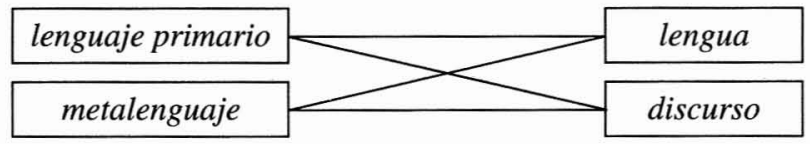


guaje y lengua del español, que constituyen una oposición inexistente en otros idiomas, como el inglés. También pertenecerán al metalenguaje de lengua en español otros elementos léxicos, como palabra, sustantivo, nombre, adverbio, hablar, decir, etc. Teóricamente, un metalenguaje de lengua, al que propongo llamar metalengua, estará constituido ante todo por un léxico especial, el referente a realidades lingüísticas, así como, según propone el propio Coseriu ${ }^{6}$, por una gramática también particular; creemos, no obstante, que este no es el caso del español, cuya metalengua está constituida únicamente por una parte del léxico, esto es, por un metaléxico, opinión de la que se muestra partidario asimismo $\mathrm{S}$. Gutiérrez $z^{7}$. Ahora bien, como también sugiere Coseriu, en este metaléxico o léxico metalingüístico hay que distinguir a su vez entre lo que podríamos llamar léxico metalingüístico tradicional frente al léxico metalingüístico terminológico, esto es, el empleado por la Lingüística. La distinción, aunque teóricamente clara, no resulta muy fácil en la práctica: habría que señalar criterios de distinción más específicos.

Volviendo a la primera distinción, correspondería al metalenguaje de discurso ante todo el especial uso que las palabras -o cualquier otro elemento del lenguaje primario o de la propia metalengua- tienen de representarse a sí mismas, como es el caso de, por ejemplo,

'Casa' tiene cuatro letras

'Juan' es un nombre horrible

'Palabra' se escribe con $b$

Así pues, metalenguaje y lenguaje primario, por una parte, y lengua y discurso, por otra, constituyen dos niveles lingüísticos diferentes, que se entrecruzan.

6 Cfr. E. Coseriu (1981: 295).

7 Cfr. S. Gutiérrez (1997: 388). Realmente el ejemplo del uso especial del artículo en español, citado por el lingüista rumano, creemos que no difiere del correspondiente a los nombres propios: realmente las palabras de la lengua utilizadas metalingüísticamente tienen, en este sentido, un comportamiento similar al de los nombres propios. Así, en

\section{Para es una preposición}

para no lleva artículo por la misma razón que éste no aparece, por ejemplo, en

Nicolás es médico. 
donde casa, Juan y el vocablo metalingüístico palabra aparecen como vocablos citados, no en su uso normal, esto es, en el relativo a expresiones del lenguaje primario del tipo

Mi casa tiene cuatro ventanas

Juan es horrible.

Corresponden estos dos usos a lo que los lógicos han llamado, respectivamente, mención y uso, o también, ya en la Edad Media, suppositio materialis y suppositio formalis. Otros, de un modo paralelo, prefieren hablar, respectivamente, de metalengua y lengua objeto.

Es evidente que cualquier elemento lingüístico, sea o no léxico, pertenezca al lenguaje hablado o escrito, puede utilizarse como representante de sí mismo, esto es, metalingüísticamente; por ejemplo, en

El prefijo in- indica negación

La letra $b$ se llama be

El signo? de interrogación

etc. Aunque se trata, evidentemente, de elementos metalingüísticos al igual que palabra, lenguaje, lengua, etc., poseen sin embargo un status muy diferente: en primer lugar y ante todo, mientras estos últimos constituyen paradigmas, esto es, están estructurados y, por lo tanto, pertenecen al sistema lingüístico (forman parte, como hemos visto, de una metalengua o metalenguaje de lengua), en este último caso se trata de elementos - no necesariamente palabras o unidades léxicas y ni siquiera a veces pertenecientes a la propia lengua- que no constituyen, contra el parecer de Rey-Debove, quien los introduce en el metaléxico, ninguna estructura paradigmática; responden a un uso, perteneciente, por tanto, al puro discurso. Y ese status diferente se muestra además en que las palabras pertenecientes a la metalengua o metalenguaje de lengua son, a su vez, susceptibles de ese mismo uso metalingüístico en el discurso, según hemos podido observar en uno de los ejemplos anteriores o en otros similares como éstos:

Los términos 'sujeto' y 'predicado' han sido tomados de la Lógica 'Sustantivo' pertenece al género masculino. 
Nos encontramos, por consiguiente, ante dos niveles metalingüísticos completamente distintos: el de la lengua y el del discurso. Porque, aunque parezca paradójico, lo que llamamos metalengua no es más que una parcela de la propia lengua entendida como sistema, básicamente un léxico especial cuya única razón de ser es el propio lenguaje, al que se refiere como una parcela más de la realidad.

Pero refiriéndonos al metalenguaje de discurso o, como prefieren llamarle otros, metadiscurso ${ }^{8}$, conviene añadir, por otro lado, que éste no se reduce, según podría deducirse de las observaciones de Coseriu, al mero "uso metalingüístico" de cualquier elemento perteneciente al lenguaje primario (o incluso al metalenguaje de lengua), sino que es necesario incluir en él lo que yo propongo llamar enunciado reflexivo o metalingüístico, constituido por aquel discurso o fragmento de discurso cuyo objeto o razón de ser no es otro que describir desde algún punto de vista una lengua o algún elemento de la misma. Así, serán enunciados metalingüísticos, por ejemplo,

El español es una lengua románica

El verbo concuerda con el sujeto en número y persona.

Cabe señalar, por otro lado, que un enunciado de este tipo puede estar constituido por elementos pertenecientes al metalenguaje de lengua (lengua, verbo, sujeto...), junto a otros pertenecientes al lenguaje primario (es, el, con, románico) o, incluso, en uso metalingüístico, como sería el caso de caballero y caballo en

Caballero deriva de caballo.

Así pues, todas las distinciones que hemos establecido hasta aquí pueden resumirse en el siguiente cuadro:

8 Así, por ejemplo, J. Dubois et alii (1979, s. v.). A nosotros, sin embargo, nos parece más apropiado considerar como metadiscurso a todo discurso que se ocupa o se refiere al propio discurso, y en el que, paralelamente, existirá un "uso metadiscursivo" junto a un "enunciado metadiscursivo"; así, en

"Lo bueno si breve dos veces bueno" es una frase de Gracián constituirá un enunciado metadiscursivo, puesto que se refiere al discurso delimitado por comillas, el cual está aquí tomado en uso metadiscursivo (es un discurso citado). 
Lenguaje primario
Metalenguaje $\left\{\begin{array}{l}\text { Metalenguaje de lengua } \\ \text { o metalengua }\end{array}<\begin{array}{l}\text { Léxico metalingüístico } \\ \text { tradicional } \\ \text { Léxico metalingüístico } \\ \text { terminológico }\end{array}\right.$
Metalengaje de discurso < Uso metalingüístico
Enunciado metalingüístico

Hay que tener en cuenta, por lo demás, que la utilización tanto del léxico metalingüístico o metaléxico como del perteneciente al lenguaje primario en uso metalingüístico es independiente del carácter a su vez metalingüístico del enunciado o enunciados en que aparecen, como lo demuestran expresiones de este tipo:

Alfonsito odia los verbos irregulares

Se despidió con un brusco adiós,

donde, por una parte, la palabra metalingüística verbo y, por otra, el vocablo mencionado -o en uso metalingüístico-adiós se insertan en realidad en sendos enunciados no reflexivos $\mathrm{y}$, por lo tanto, carentes de valor metalingüístico.

\subsection{El USO METALINGÜÍSTICO}

Centrando ahora nuestra atención en el metalenguaje de discurso y más concretamente en el uso metalingüístico, es frecuente interpretarlo como la utilización de una palabra o elemento lingüístico que o bien actuaría como nombre de sí mismo o, también, como mera palabra o pura forma desposeída de todo significado, interpretaciones que, a nuestro modo de ver, resultan inadecuadas por varias razones:

a) En primer lugar si, por ejemplo, una palabra como para se tomase como nombre de la preposición correspondiente, ocurriría que en nuestra lengua existirían dos signos homónimos para: uno correspondiente al uso de la preposición en el lenguaje primario (por ejemplo, en 
y otro al uso metalingüístico (así, en

Para acompaña a algunos complementos del verbo).

Es decir, junto a la preposición para existiría un sustantivo homónimo precisamente con la función de nombrar esa misma preposición. Pero notemos que por esta regla de tres todo vocablo formaría parte no solo de la lengua sino de la metalengua, al poseer siempre una contrapartida metalingüística, circunstancia que, lógicamente, duplicaría el léxico de la lengua. Y no solo esto, pues incluso las palabras de otros idiomas y otros muchos elementos que ni siquiera son palabras, al ser asimismo susceptibles de uso metalingüístico o de mención, formarían también parte del metaléxico, el cual vendría a ser así algo ilimitado y, por ello, absolutamente incontrolable. Y tal es, por cierto, la idea defendida por Rey-Debove, quien llega a postular la pertenencia de esos presuntos homónimos -“autónimos" les llama la autora francesa- al metaléxico, del que formarían parte al lado de vocablos como palabra, decir, nombre o adjetivo, que serían en todo caso palabras metalingüísticas stricto $s e n s u^{9}$. Y en esta misma línea se ha llegado a hablar alguna vez de una metábasis o transposición a la función de sustantivos, que caracterizaría a todas las palabras ${ }^{10}$. Nada más lejos, sin embargo, de la realidad, pues no hay duda de que en cualquiera de los dos casos nos encontramos ante la misma palabra, aunque -eso sí- en usos diferentes.

b) Cabe, no obstante, preguntarnos si en el uso metalingüístico no se tratará más bien del simple significante, o lo que es lo mismo, de la palabra desposeída de su significado. Y nuestra conclusión es que esto tampoco es aceptable, pues, si bien para algunos, por ejemplo, la palabra casa en un enunciado como

\section{Casa está compuesto por dos silabas}

estaría tomada exclusivamente en su significante, ello no es así ni mucho menos. Si bien nos fijamos, lo que aquí se refiere al significante es

9 Cfr. J. Rey-Debove (1978: 29).

10 Así S. Gutiérrez Ordóñez (1997: 392). 
la pura predicación, pero no el sujeto de esa predicación, que es el mismo que aparece en este otro enunciado:

\section{Casa es parasinónimo de edificio}

cuya predicación se refiere, en cambio, al significado. Así pues, todo esto quiere decir que, en su uso metalingüístico, cualquier palabra o elemento significativo está tomado en todas sus dimensiones, esto es, como tal signo o palabra y, por lo tanto, la predicación, que forma parte del enunciado metalingüístico, puede referirse, por ello, a cualquiera de esas dimensiones o aspectos.

c) Pero a todo esto hay que añadir todavía más: este signo o palabra - o cualquier otro elemento en uso metalingüístico- está utilizado como representante de sí mismo, cosa que no quiere decir, contra lo que suele aceptarse (sobre todo por parte de quienes se han ocupado del asunto desde presupuestos lógicos), que sean nombres o signos de sí mismos. Nótese que, si ello fuera cierto, nos veríamos obligados a entrar en una especie de recursivididad que implicaría la existencia de una serie infinita de niveles metalingüísticos, toda vez que, por otro lado, el nombre de una palabra podría a su vez utilizarse como mención de sí mismo, con lo que tendríamos un uso metalingüístico de otro metalingüístico y así sucesivamente; de ese modo podría generarse una cadena infinita de enunciados:

Para es una preposición > Para es el nombre de la preposición para; El nombre para de la preposición para es invariable $>$ Para es el nombre del nombre para correspondiente a la preposición para...

etc. El representarse una palabra o elemento lingüístico a sí mismo en un contexto cualquiera no significa ni mucho menos que sea signo de sí mismo, sino que tal objeto, por el mero hecho de tener naturaleza lingüística (con expresión fónica o gráfica), no necesita transformación semiótica alguna: se tomará como tal objeto y punto. Si bien nos fijamos, sólo una realidad no lingüística necesita de un elemento lingüístico o signo que lo represente para poder formar parte de un texto; pero un ente o realidad perteneciente al lenguaje -e incluso no lingüístico, pero de naturaleza fónica o gráfica- no necesita de otro para funcionar 
como elemento de ese texto: es autosuficiente y puede, por tanto, emplearse directamente. Así se explica, por ejemplo, que en un enunciado como el siguiente, que además de lingüístico es también gráfico, tenga sentido escribir esto:

Be es el nombre de $b$,

donde, obviamente, $b$ está tomada como objeto o entidad en sí mismo; no como representante de la letra correspondiente, sino como la propia letra. Insistimos en que aquí la representación directa es posible porque se trata de una realidad gráfica en un texto asimismo gráfico, y distinta claramente de su correspondiente nombre. Por eso carecería de sentido su lectura, esto es, su versión oral, donde la letra en cuestión necesita de una representación fónica, esto es, expresarse mediante la versión oral de su correspondiente nombre, produciéndose así una expresión tautológica equivalente a su vez a este otro enunciado escrito:

Be es el nombre de be,

$\mathrm{y}$, correspondientemente, tendríamos:

Madrid es el nombre de Madrid.

Para es el nombre de la preposición para,

cosa que, además, en este último caso no sería verdad, puesto que para no es ningún nombre, sino que por ser un objeto lingüístico puede desempeñar en un enunciado los mismos papeles que un sustantivo, que no es lo mismo.

En conclusión, las palabras -como cualquier otro elemento de naturaleza fónica o gráfica- pueden usarse metalingüísticamente, esto es, como tales objetos, con independencia de las posibles funciones para las que han sido creadas. Al fin y al cabo las palabras, constituyentes de palabra, fonemas o segmentos de la cadena hablada en general son también objetos de la realidad y, como tales, también pueden ser mentados en el discurso, pero con la peculiaridad de que, para esto, no se se necesita echar mano de elementos vicarios o signos que los representen -como ocurre con las otras realidades-, sino que ellos son autosuficientes, se representan directamente a sí mismos. 


\subsection{Otros usos METALINGÜÍsTICOS}

Para distinguir el uso metalingüístico del lingüístico en una palabra se ha acudido con frecuencia al convencionalismo gráfico de escribirla en el primer caso entre comillas o comillas sencillas, de modo que de esa forma, por ejemplo, el significado de

Me disgusta 'Juan'

se interprete de manera distinta a

Me disgusta Juan".

Un procedimiento, sin embargo, quizás más adecuado consistirá en escribir dicha palabra en una letra diferente (por ejemplo, en cursiva, como viene siendo habitual en la literatura lingüística en la mención de palabras) y dejar las comillas sencillas para la indicación de los puros significados.

Pero precisamente esta última observación me lleva a la necesidad de ampliar el concepto de "uso metalingüístico", que, a mi modo de ver, no se reduce a la exclusiva mención del signo o suppositio materialis, como generalmente se viene aceptando. Sea, por ejemplo, el enunciado

La palabra perro -escrita <perro > y pronunciada [péro]- significa 'perro',

donde observamos que pese a que el segmento gráfico "perro" se repite varias veces, en ninguno de los casos tiene el mismo valor, sin que, por otro lado, coincida en ninguno de ellos con el que posee en el lenguaje primario, como sería, por ejemplo, el caso en este otro contexto:

El perro es el mejor amigo del hombre.

Esto significa que en el texto en cuestión nos encontramos en realidad ante usos metalingüísticos diversos. Y así es efectivamente: mientras

11 Cfr. J. Lyons (1980: 9). 
en su primera aparición perro responde claramente a la mención directa de que acabamos de ocuparnos, esto es, coinciden signo y referente, en la segunda y tercera representa únicamente al significante (gráfico y fónico, respectivamente) y, finalmente, en la cuarta alude tan solo el significado de dicha palabra. Gráficamente,

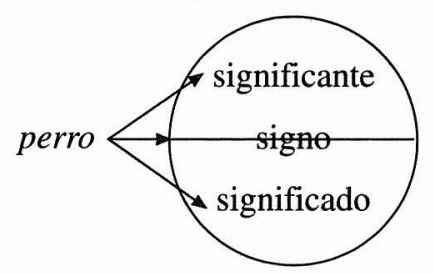

Para demostrar que se trata de usos diferentes, notemos que, en el supuesto de que las palabras del español estuviesen numeradas y a perro le correspondiese el número 458, éste tan solo podría sustituirla en la primera aparición ${ }^{12}$, por lo que podría decirse

La palabra 458 tiene el significante < perro $>$ y el significado 'perro'.

Por su parte 'perro' como representante del significado podría sustituirse aquí por 'can' o por una expresión del tipo 'mamífero doméstico de la familia de los cánidos', esto es, por una definición lexicográfica. Y asimismo, como representante del significante gráfico, podrían usarse distintos tipos o tamaños de letras o incluso sistemas gráficos diversos, etc.

En resumidas cuentas, en todos estos casos el elemento lingüístico "perro" es interpretado y categorizado como signo (palabra) perteneciente a nuestro idioma, como conjunto de letras o sonidos que en el nivel del significante constituyen ese signo y, finalmente, como mero significado, el asimismo correspondiente a ese signo. Decimos que nos hallamos ante usos metalingüísticos porque en todos los casos el segmento en cuestión se enfoca como realidad lingüística, bien es verdad que en la tercera y cuarta mención del ejemplo inicial, por tratarse de 
realidades no gráficas, hay que acudir, respectivamente, a una transcripción que coincide con la representación gráfica del signo, esto es, se acude a procedimientos de representación indirecta. Pues bien, esto nos lleva a postular la existencia de dos tipos de uso metalingüístico, unos de representación directa (el uso metalingüístico que podemos llamar "de signo" y de "significante"), junto a usos metalingüísticos de representación indirecta, esto es, mediante a su vez un signo coincidente con el inicial (el uso metalingüístico de significado). Lógicamente, el uso metalingüístico de significante gráfico sería directo en textos escritos, y el de significante fónico lo sería en los orales.

\section{EL METALENGUAJE EN LEXICOGRAFÍA}

Precisamente la distinción entre uso metalingüístico de signo y uso metalingüistico de significado parece recordarnos la distinción, ya antes aludida, propuesta hace años por J. Rey Debove, y que ha sido en general aceptada en la lexicografía teórica posterior, a propósito de las definiciones lexicográficas ${ }^{13}$, entre primera metalengua $\mathrm{o}$, como prefiere llamarla M. Seco, primer enunciado o metalengua de contenido, y segunda metalengua o, según este último, segundo enunciado o metalengua de signo $^{14}$. La distinción, sin embargo, no coincide exactamente con la nuestra, ya que, mientras nosotros con la expresión "uso metalingüístico de signo" nos referimos a la mención directa de la palabra (por ejemplo el correspondiente a las entradas del diccionario), los autores anteriores -y más concretamente M. Seco- llaman "metalengua de signo" a las indicaciones que, dentro o fuera de la definición, atañen a aspectos que no tienen que ver con el significado; así, por ejemplo, en el caso de

lleno, na [...]. Dicho de personas, un poco gordo

que encontramos en el DRAE, el definiens estaría constituido, siguiendo a Rey-Debove y a M. Seco, por dos partes: una que pertenecería a la

13 Cfr. J. Rey-Debove (1967: 143).

14 Cfr. M. Seco (1987: 15 y ss.). 
metalengua de signo o segunda metalengua y representada por lo que aparece en letra redonda, y otra, en cursiva, correspondiente a la metalengua de contenido. Así pues, tan solo nuestro "uso metalingüístico de significado" parece coincidir con la primera metalengua, primer enunciado o metalengua de contenido de los antedichos autores; pero esta apreciación no es del todo exacta, porque -recordémoslo-nuestro "uso metalingüístico de significado" corresponde más bien a la palabra utilizada para representar su propio significado: en el ejemplo anterior sería, por tanto, 'lleno'; pero no este mismo significado expresado mediante un sinónimo o definición, ya que en este caso no existiría el mismo tipo de reflexividad lingüística ${ }^{15}$. Como veremos más adelante, la distinción de Rey-Debove no apunta en realidad a distintas metalenguas, ni siquiera a diferentes usos metalingüísticos, sino a la utilización de la función metalingüística en diversos niveles o instancias del enunciado lexicográfico.

\subsection{NIVELES DE REFLEXIVIDAD Y TIPOS BÁSICOS DE DISCURSO METALINGÜÍSTICO}

Desde luego, no cabe duda de que, en comparación con otros tipos de estudios sobre el lenguaje, es en los diccionarios donde la función metalingüística adquiere una particular complejidad e importancia, circunstancia que justifica que centremos aquí nuestra atención en ella, habida cuenta, además, de que se trata de una cuestión que todavía no ha sido estudiada, a mi juicio, en la profundidad y extensión que se merece.

Para empezar, es evidente que un diccionario, por cuanto que constituye un estudio del léxico, representa, como cualquier otra obra sobre objeto lingüístico, una consideración del lenguaje por medio del lenguaje $y$, por lo tanto, responde claramente a la función metalingüística. Se trata de un estudio "reflexivo" en la medida en que el objeto de estu-

15 Solo secundariamente podría pensarse en un "uso metalinlingüístico", pero del sinónimo o definición, no del definido o entrada lexicográfica. Sobre esto volveremos luego (vid. § 2.5). 
dio se identifica con el instrumento empleado para dicho estudio, identificación que por cierto puede llevarse a efecto en tres estadios o niveles diferentes: en el concreto de la palabra, en el particular de la lengua o en el general del lenguaje. Pertenecerán al primero las entradas, constituidas por palabras que se refieren a sí mismas, esto es, en uso metalingüístico; en el segundo, por su parte, como ocurre en el diccionario monolingüe, objeto de estudio e instrumento se identifican por corresponder a la misma lengua, mientras que en el tercero -así en el diccionario bilingüe- se trata de sistemas lingüísticos diferentes, por lo que la reflexividad se produce en un nivel más general, en el del lenguaje.

Con frecuencia, por cierto, tiende a llamarse metalengua o metalenguaje exclusivamente al sistema que desempeña el papel de instrumento, lo cual es inexacto en dos sentidos:

a) Porque, después de lo que llevamos dicho, resulta absolutamente inapropiado hablar aquí de "metalengua", a no ser que dicho sistema instrumental viniera representado por un lenguaje artificial, inventado ex profeso para la descripción lexicográfica, lo que constituiría en todo caso un procedimiento posible, pero inhabitual en Lexicografía, cuyas descripciones o explicaciones, por razones prácticas obvias, se vienen haciendo por medio de una lengua natural. En todo caso, lo que ofrece carácter metalingüístico no es, según ya quedó suficientemente aclarado, el sistema empleado como instrumento, sino el discurso creado a partir de él.

b) Pero hay que tener en cuenta, por otro lado, que no solo la lengua utilizada como instrumento da lugar a un discurso metalingüístico. Repetimos que también la que sirve de objeto de estudio pertenece al mismo tipo de discurso en la medida en que, como acabamos de observar, las entradas del diccionario están tomadas en él como representantes de sí mismas, esto es, como palabras o unidades léxicas en general.

En resumidas cuentas, todo esto nos lleva a afirmar que, al menos en principio, cada artículo lexicográfico -y en definitiva la totalidad del diccionario- no viene a ser más que un texto o discurso de carácter metalingüístico: lo que en él se contiene no es otra cosa que un conjun- 
to de informaciones sobre diversos aspectos de las unidades lingüísticas que componen una lengua. La lengua objeto de estudio, representada en la macroestructura por todas las entradas, se halla mencionada, por lo que corresponde claramente a un "uso metalingüístico", y, por otro lado, las diversas informaciones, presentes en la microestructura y expresadas mediante la lengua instrumental, constituyen por su parte verdaderos enunciados metalingüísticos.

\subsection{METALENGUAJE Y FUNCIONES INFORMATIVAS}

Alguna vez se ha dicho que un artículo lexicográfico no viene a ser otra cosa que una oración cuyo sujeto es la entrada, y el predicado o predicados, cada una de las informaciones registradas en dicho artículo ${ }^{16}$, lo que equivaldría a decir que lo que aquí llamamos "enunciado metalingüístico" tendría una función sintáctica de predicado, frente a las unidades léxicas estudiadas, que precisamente, por corresponder a "usos metalingüísticos" -están tomadas como ellas mismas-, funcionarían como sujetos de esa predicación. La afirmación podría aceptarse, aunque ello depende, lógicamente, de lo que se entienda por 'oración': se trataría de todas formas de una oración sui géneris, dado que, por una parte, el sujeto se hallaría totalmente desligado del predicado, al constituir ambos unidades tonales independientes (separación gráficamente representada por un punto), y, por otro, no siempre el predicado constaría de un verbo concordado con dicho sujeto, verbo que, si bien sería fácilmente restituible en unos casos, no lo sería en cambio en otros; así, mientras en

frío, a [...]. Aplícase a los cuerpos cuya temperatura es muy inferior a la ordinaria del ambiente

la forma verbal aplicase está sin duda referido a la palabra frío, sintácticamente sujeto, no existe ningún verbo equivalente en

excarcelable. adj. Que puede ser excarcelado

16 Cfr. J. y Cl. Dubois (1971: 39) e I. Ahumada (1989: 46). 
donde nos encontramos con dos predicaciones: la primera representada por la marca de categorización "adj[etivo]", y la segunda por la definición; pero notemos que, si bien en el primer caso se podría pensar en un verbo elíptico de tipo atributivo (excarcelable [es] adjetivo), no sería posible postular tal elipsis en el segundo caso, pues una oración de relativo, como es ésta, no puede de hecho actuar como predicado nominal: sería inaceptable

*excarcelable es que puede ser excarcelado.

Más que de oración, a propósito del artículo lexicográfico, creemos que sería preferible hablar de un texto o enunciado metalingüístico especial constituido por dos elementos: un componente temático, representado por la entrada, junto a una serie de componentes remáticos, constituidos por cada una de las diversas informaciones contenidas en el artículo, informaciones que, evidentemente, no hay que identificar exclusivamente con las definiciones, sino con cualquier tipo de indicación acerca de la naturaleza, características, usos y funcionamiento de la entrada. No hay que olvidar, efectivamente, que un diccionario está estructurado de manera que el usuario a partir de la nomenclatura o conjunto de entradas, concebidas como información conocida, pueda informarse acerca de diversos aspectos que, presumiblemente, le puedan ser desconocidos y, por lo tanto, se entienden como información nueva. Un artículo lexicográfico en verdad no es más que un conjunto de respuestas a una serie de preguntas que el usuario del diccionario puede plantearse acerca de una misma unidad léxica, la que aparece como entrada.

Pero a propósito de esta última conviene notar que en la práctica su papel no se reduce exclusivamente a servir de tema en el enunciado metalingüístico, sino que, al mismo tiempo, puede aportar alguna información nueva al usuario, lo que significa que posee asimismo un cierto valor remático: ante todo su presencia en el diccionario informa al menos de su existencia como unidad léxica de la lengua objeto de estudio y, en segundo lugar, puede dar información acerca de las características del significante, que en realidad es lo que aparece directamente representado. Estas dos funciones son bien claras cuando, por 
ejemplo, consultamos un diccionario porque dudamos de la aceptación o corrección de un determinado vocablo, o porque tenemos dudas acerca de su ortografía. Así pues, por ejemplo, la simple entrada hilo contenida en el correspondiente artículo de un diccionario nos informará por sí sola, entre otras cosas, de que se escribe con hache o de que es una palabra del léxico español. Concluyendo, digamos que las entradas constituyen de hecho enunciados por sí mismas, los cuales, utilizando estructuras oracionales, podrían expresarse poco más o menos asi ${ }^{17}$ :

Hilo es un vocablo del español

Hilo se escribe con $h$

Hilo tiene cuatro letras

Hilo es una palabra llana,

etc. Se trata, no obstante, como puede verse, de enunciados sintácticamente no explícitos, consistentes en puras inferencias realizadas a partir de la entrada misma, cuya función es, repetimos, esencialemente temática correspondiendo, por otro lado, como hemos visto, a un "uso metalingüístico" del vocablo o elemento léxico en cuestión. La entrada, en definitiva, no es otra cosa que el tema del artículo lexicográfico, cuyo cuerpo está a su vez integrado por toda una serie de enunciados lexicográficos, cada uno de los cuales representa un componente remático o informativo.

\subsection{TIPOS DE ENUNCIADO EN EL DICCIONARIO}

Así pues, son estos enunciados lexicográficos, sintácticamente explícitos, los que representan más propiamente el componente informativo del diccionario, y poseen además en principio carácter metalingüístico. Y digo "en principio", porque, en la práctica lexicográfica tradicional y habitual, no todos los datos que de hecho aparecen en los

17 Esto sin contar con que la adopción de un determinado tipo o tamaño de letra en la entrada puede utilizarse para informar acerca de alguna característica de la entrada. Así, en la primera edición del DuE de $\mathrm{M}^{\mathrm{a}}$ Moliner, una entrada en un cuerpo menor significa que se trata de un vocablo caído en desuso. 
artículos de un diccionario se refieren siempre a la entrada entendida como unidad lingüística. Así, por ejemplo, en el siguiente ejemplo tomado del DRAE

\section{escarapela [...] f. Divisa compuesta de cintas por lo general de va- rios colores, fruncidas o formando lazadas alrededor de un punto. Como distintivo, se coloca en el sombrero, morrión, etc. Se usa tam- bién como adorno}

es evidente que lo que se coloca en el sombrero o se usa como adorno no es la palabra escarapela, sino el objeto designado por ella, y, por lo tanto, las explicaciones que aquí hemos puesto en cursiva no son en absoluto metalingüísticas, pues se hallan referidas al objeto representado por la palabra. Ahora bien, esto quiere decir que de los enunciados contenidos en un artículo lexicográfico no todos tienen carácter metalingüístico; unos lo tendrán y otros no, de manera que, si les llamamos enunciados lexicográficos, habrá que distinguir dos subtipos diferentes: enunciados lexicográficos metalingüísticos, los que se hallan referidos a la entrada entendida como unidad léxica, junto a los meramente lingüísticos, aquellos que, por el contrario, aluden al referente o realidad indicada por la palabra-entrada. Por consiguiente, en el ejemplo anterior lo indicado en cursiva corresponde, como puede verse, a dos enunciados meramente lingüísticos, y sólo lo que aparece en redonda corresponderá a un enunciado propiamente metalingüístico, aun cuando la definición en él expresada puede aludir tanto a la palabra-entrada como al objeto que actúa como referente de ella.

Y precisamente esta última observación nos sirve para enlazar con el planteamiento de una cuestión que, a propósito del carácter metalingüístico del discurso lexicográfico, parece fundamental: ¿Hasta qué punto las definiciones contenidas en los diccionarios son realmente definiciones de las palabras o más bien de las realidades representadas por ellas? Desde luego si nos fijamos en lo que ocurre en la práctica lexicográfica tradicional, fácilmente podremos constatar que en la formulación de definiciones se prescinde sistemáticamente de la distinción - tan fundamental hoy en la semántica moderna- entre significado y designación, no siendo por otro lado infrecuente la -sin duda más 
grave- confusión entre signo y cosa, tal como ocurre normalmente en el uso corriente de la lengua. Retomando el ejemplo anterior de escarapela, pueden fácilmente observarse todas esas confusiones: por una parte notemos que la entrada viene dada en tanto palabra, puesto que se halla clasificada como femenina, pero la definición propiamente dicha parece más bien referida a la realidad representada por la entrada, cosa que resulta del todo evidente al leer las explicaciones complementarias a que acabamos de referirnos. Ahora bien, siendo esto así, parece lógico pensar que tan solo se dará verdadera función metalingüística en las definiciones referidas a las palabras-entrada, en tanto que carecerán de esa función la definiciones enciclopédicas o referidas a las cosas, observación que por cierto parece contradecirse con lo que decíamos al final del párrafo anterior.

La contradicción es, sin embargo, tan solo aparente, ya que una cosa es el enunciado definicional y otra el enunciado lexicográfico, del que en realidad aquélla forma parte. Lo que queríamos decir antes es que un enunciado lexicográfico de carácter metalingüístico puede contener una definición que a su vez puede ser o no metalingüística. Y es precisamente a estos dos tipos de enunciado a los que sin duda apuntan J. Rey-Debove y M. Seco con la distinción, antes aludida, entre primer enunciado o metalengua de contenido por una parte, y segundo enunciado o metalengua de signo por otra; pero con la diferencia de que para nosotros no se trata de dos metalenguajes distintos, sino, simplemente, de dos instancias discursivas en que puede llevarse a cabo la función metalingüística. Un enunciado lexicográfico es metalingüístico por el mero hecho de estar referido a una palabra-entrada, de la que indica alguna característica; pero como cualquier enunciado de ese tipo puede a su vez estar constituido por elementos que, asimismo, pueden ser o no metalingüísticos desde algún punto de vista. Supongamos, por ejemplo, el enunciado, tomado del Diccionario de Autoridades,

apagar. Metaphoricamente significa destruir y acabar con alguna cosa

cuyo carácter metalingüístico es evidente por tratarse de una predicación de la palabra apagar; pero notemos que, a su vez, los componen- 
tes metafóricamente y significar pertenecen al léxico metalingüístico del español (forman parte del metalenguaje de lengua), y, por otro lado, la definición, representada por el enunciado destruir y acabar con alguna cosa, por referirse al significado de apagar, posee asimismo función metalingüística. He aquí, pues, un enunciado definicional metalingüístico dentro de un enunciado lexicográfico, más amplio, con función a su vez metalingüística.

\subsection{ESTRUCTURA DEL ENUNCIADO LEXICOGRÁFICO}

Esto supuesto, un enunciado lexicográfico que contenga una definición consiste en una estructura sintáctica representada por una oración cuyo verbo -junto a veces con algunos elementos que le rodean-sirve de enlace entre, por una parte, la entrada (sobreentendida cuando actúa como sujeto, o representada por un anafórico si desempeña otra función sintáctica) y, por otro lado, la definición o referente de la entrada. Así, partiendo del ejemplo anterior, tendremos el siguiente esquema:

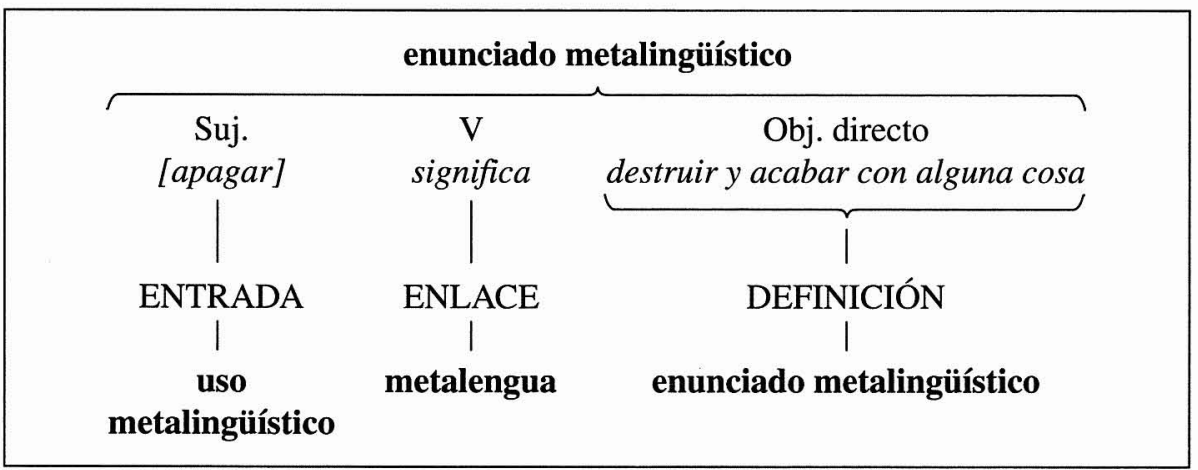

Un tipo a este respecto muy frecuente de enunciado lexicográfico en los diccionarios es por cierto el que comienza por expresiones como dicese de, aplicase a, que representan precisamente el enlace entre la palabra-entrada y el enunciado definicional, que en este caso actúa como complemento preposicional y carece, por otro lado, de función metalingüística por aludir al referente de la entrada y no a su significado. Considérense, entre los muchísimos ejemplos que podrían citarse, los siguientes: 
documental [...]. Dícese de las películas cinematográficas o programas de televisión que representan, con propósito meramente informativo, hechos, escenas, experimentos, etc.

dominante [...]. Aplicase a la persona que quiere avasallar a otras, y a la que no sufre que se le opongan o la contradigan.

Por lo que se refiere al enlace, hay que observar que no siempre viene representado exclusivamente por un verbo, sino por toda una frase constituida por ese verbo y un conjunto de palabras incidentes con el mismo, entre las cuales puede estar incluso el sujeto de la oración, cuando éste no coincide con la entrada. Así, por ejemplo, en el siguiente enunciado tomado del DRAE

doblón [...]. El vulgo llamó así, desde los Reyes Católicos, al excelente mayor, que tenía el peso de dos castellanos o doblas.

la función de enlace corresponde al texto que transcribimos en cursiva, y la entrada está representada anafóricamente por así. Por otro lado, aunque frecuentemente dicho verbo corresponde al léxico metalingüístico, esto es, al metalenguaje de lengua -como ocurre en los ejemplos anteriores-, ello no siempre es así, sobre todo cuando el enunciado lexicográfico se expresa sintácticamente mediante una oración atributiva, en cuyo caso no aparece el verbo; la pertenencia al léxico metalingüístico corresponderá entonces al núcleo del predicado. Así, en este otro enunciado, tomado asimismo del DRAE,

liceo [...]. Nombre de ciertas sociedades literarias o de recreo.

el enunciado lexicográfico consiste en un predicado nominal referido al sujeto representado por la entrada, en que a su vez está integrado el enunciado definicional (en letra redonda) y el enlace (en cursiva), cuyo componente nombre pertenece a la metalengua.

No infrecuentemente un enunciado lexicográfico incluye otro enunciado lexicográfico, en el que a su vez se inserta la definición. Es lo que ocurre, por ejemplo, en

anti- [...] pref. que significa "opuesto" o "con propiedades contrarias" 
consistente, en primer lugar, en una oración atributiva (anti- es un prefijo...), pero a su vez significa "opuesto"... constituye en realidad otro enunciado, referido sintácticamente mediante adjetivación relativa a prefijo, pero que en realidad podría aplicarse directamente a la entrada: anti- significa "opuesto"... Hay, por lo tanto, aquí un doble enlace, representado básicamente por las palabras de la metalengua prefijo y significar.

A lo dicho conviene añadir que, por regla general, el enlace puede tormarse además como marca del carácter metalingüístico o no metalingüístico del enunciado definicional. Lógicamente, si el enlace está constituido por el verbo significar u otro equivalente, es claro que la definición se refiere al contenido o significado de la entrada y, por lo tanto, tendrá carácter metalingüístico; pero no ocurrirá lo mismo cuando dicho verbo es decir, aplicar, llamar y otra expresión semejante, en cuyo caso la definición que aparece seguidamente aludirá, obviamente, al referente u objeto indicado por la entrada. Por esa razón el problema surge cuando, como es práctica más habitual en la lexicografía moderna, el enunciado lexicográfico carece de enlace, como ocurre, por ejemplo, en estos enunciados:

precio [...] Valor pecuniario en que se estima una cosa

estrato [...] Nube que se presenta en forma de faja en el horizonte

madre $[\ldots]$ Hembra que ha parido

ya que, al coincidir el enunciado lexicográfico con el definicional, no sabremos a ciencia cierta si se trata de definiciones metalingüísticas, referidas al significado, o de definiciones de las cosas o referentes de la entrada. Todo dependerá de si el enlace sobreentendido viene dado por el verbo significar o, por el contrario, llamar, denominar, decirse de, etc.

\subsection{UNA ÚLTIMA PRECISIÓN Y CONCLUSIONES}

Centrándonos ahora en el enunciado definicional referido al significado, cabe todavía hacer una última precisión acerca de su carácter metalingüístico. Como hemos observado, éste surge del hecho de tener 
como referente el significado de la palabra-entrada, esto es, el poner de manifiesto un aspecto plenamente lingüístico de aquélla. Pero notemos que, al mismo tiempo, dicho enunciado está representado a su vez por un signo cuyo significado lingüístico coincide con el de la entrada -es sinónimo de ella-, lo que quiere decir que se halla asimismo referido a su propio significado. Ahora bien, esto significa que el enunciado definicional es doblemente metalingüístico, pues en primer lugar, como acabamos de observar, describe un aspecto lingüístico de la entrada, constituyendo así, como decimos, un verdadero enunciado metalingüístico, pero al mismo tiempo se halla de alguna manera referido a sí mismo, constituyendo, por tanto, lo que hemos denominado "uso metalingüístico de significado". Gráficamente, podemos representar esto de la siguiente manera:

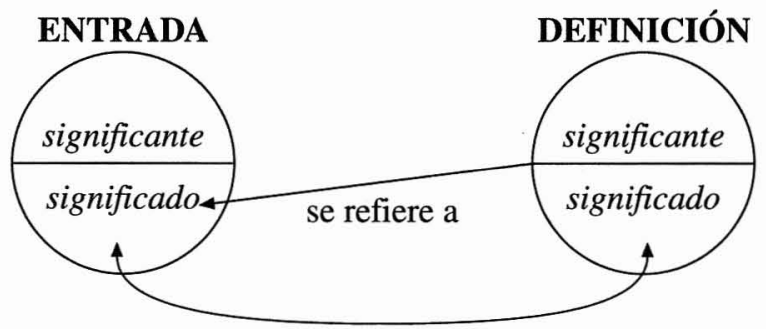

Para buscar un ejemplo sencillo, supongamos una definición de las llamadas sinonímicas, esto es, representada por una única palabra, como la siguiente, que tomamos también del DRAE:

colorín [...]. Jilguero.

Parece evidente que no solo colorín, la entrada, está usada reflexivamente, esto es, como representante de sí misma, sino también la definición, jilguero, que al referirse en primera instancia al significado de la entrada, constituyendo así el único componente de un enunciado definicional, se está en realidad refiriendo también a sí mismo, ya que se trata de su propio significado. Ello hace posible que los términos puedan invertirse pasando a definiendum el definiens y a definiens el definiendum:

Jilguero. Colorín. 
En conclusión, pues, una definición de significado puede considerarse, repetimos, doblemente metalingüística, al corresponder, por una parte, a un enunciado metalingüístico y, por otra, aunque secundariamente, a un uso metalingüístico de significado.

Así pues, para terminar, podemos resumir lo dicho acerca del metalenguaje en el diccionario, señalando, en primer lugar, que la parte informativa del artículo lexicográfico está constituida por toda una serie de afirmaciones sobre la palabra- entrada, cada una de las cuales constituye un enunciado lexicográfico, el cual, por el mero hecho de referirse a la entrada como elemento lingüístico, desempeñará, lógicamente, una función metalingüística. Tan solo enunciados, también a veces presentes en el artículo, referentes al objeto indicado por la entrada carecerán de esa función metalingüística, circunstancia que nos lleva a proponer la existencia de enunciados lexicográficos metalingüísticos junto a enunciados lexicográficos puramente lingüisticos. Por otra parte, un enunciado lexicográfico puede estar a su vez integrado por otros elementos con carácter también metalingüístico, o bien porque forman parte de la metalengua -concretamente del metaléxico-, o bien porque, a su vez, constituyen por sí mismos enunciados metalingüísticos, como ocurre con las definiciones cuando se refieren al significado de la palabra-entrada; en este último caso se puede hablar incluso, como acabamos de ver, de una doble función metalingüística. Concluyendo, todo esto no significa ni mucho menos que existan, contra lo que se viene admitiendo, dos metalenguas lexicográficas distintas, ni siquiera dos tipos de metalenguaje de discurso; en realidad cuando se apunta a esta doble posibilidad, de lo que se trata simplemente es dos niveles o estadios del discurso lexicográfico: el correspondiente, por una parte, al enunciado lexicográfico, y, por otra, al enunciado definicional, en los cuales puede llevarse a cabo, con carácter independiente, la función metalingüística. Queremos decir que las definiciones lexicográficas no siempre son metalingüísticas, aunque forman siempre parte -a veces como constituyentes explícitos únicos- de un enunciado lexicográfico que, obviamente, será metalingüístico si se halla referido a le entrada entendida como palabra o elemento perteneciente a la lengua estudiada por el diccionario. 


\section{BIBLIOGRAFÍA}

Ahumada, Ignacio (1989): Aspectos de lexicografia teórica, Granada, Universidad de Granada.

Coseriu, Eugenio (1977): Principios de semántica estructural, Madrid, Gredos.

- (1981): Lecciones de lingüistica general, Madrid, Gredos.

Dubois, J. y Cl. DuboIs (1971): Introduction à la lexicographie: le dictionnaire, Paris, Larousse.

Dubors, J. et alii (1979): Diccionario de Lingüística, Madrid, Alianza editorial.

GutiÉRrEZ OrdóÑEZ, Salvador (1997): Principios de sintaxis funcional, Madrid, Arco/Libros.

JAKOBSON, Roman (1963): Essais de linguistique générale, Paris, Minuit.

LYONS, John (1980): Semántica, Barcelona, Teide.

ReY-Debove, J. (1967): "La définition lexicographique: bases d'une typologie formelle", Travaux de Linguistique et Littérature, V/1, pp. 141-159.

_- (1978): Le metalangage. Étude linguistique du discours sur le langage, Paris, Le Robert.

SECO, Manuel (1987): "Problemas formales de la definición", en Estudios de lexicografia española, Madrid, Paraninfo, pp. 15-34. 